\title{
Palliative care; what do you really mean?
}

\section{Jessica Mackriell ${ }^{1}$, Jennifer Hunt ${ }^{2}$}

1. Palliative Care Association of Malawi (PACAM), Lilongwe, Malawi

2. African Palliative Care Association

Correspondence: Jessica Mackriell, PACAM, Email: info.pacam@gmail. com

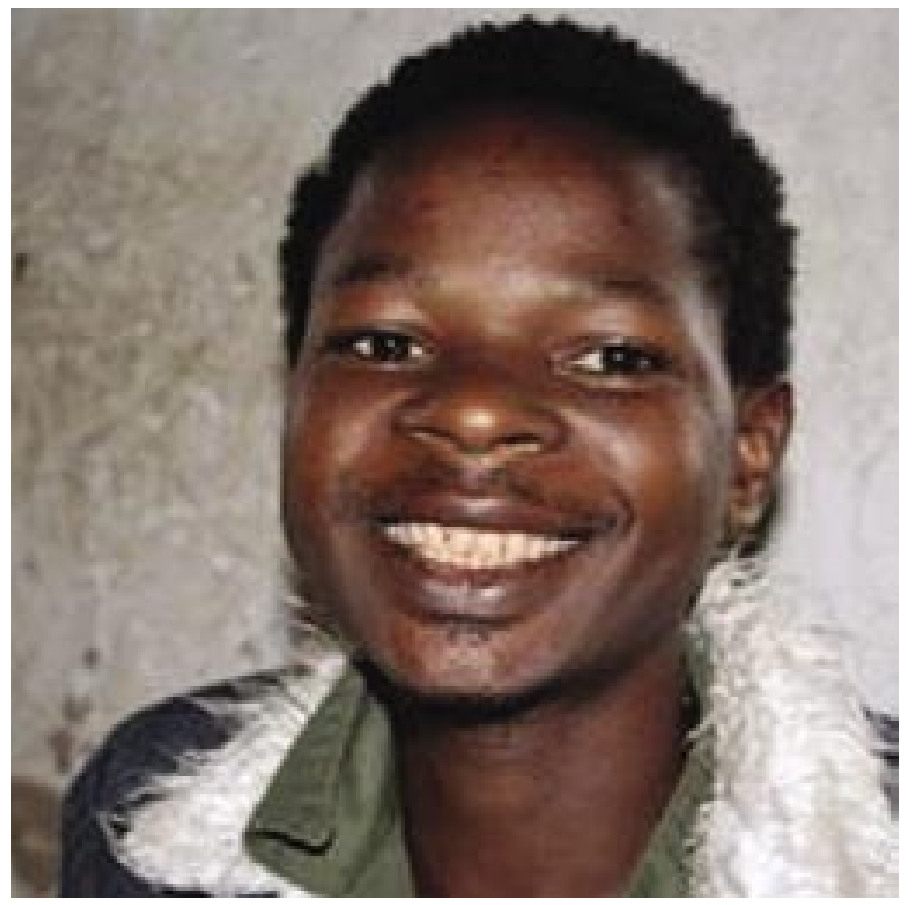

Hama Dikani - a palliative care patient in Ndirande, Blantyre

\section{Background}

'Palliative Care' was unknown in Malawi until Dr. Anne Merriman visited in 2002 and introduced the concept. HIV/ AIDS care and treatment services gradually grasped this philosophy of care, palliative care champions studied for post-graduate qualifications, health care workers gained extra skills, and a national voice for stakeholders was established in 2005 .

The Palliative Care Association of Malawi is a network of individuals and organisations working together to develop palliative care in Malawi; improving access and building quality. The National Coordinator of the Association has personally visited district hospitals to raise awareness and inspire care providers. These sensitisation efforts have been supported by training and networking. A systematic appraisal has yet to be done to assess the impact of these strategies. ${ }^{1}$

'Palliative care' is now mentioned in a number of fora in Malawi; from policy development meetings to implementation strategies, and in medical journals. Donor priorities have helped raise the profile. Since May 2008, there has been a Ministry of Health 'Introduction to Palliative Care' training course for health care workers. ${ }^{2}$ There is a palliative care component in the ARV training for health workers and in the Community Home Based Care (CHBC) training for volunteers. Palliative care learning objectives have been incorporated into the undergraduate medical curriculum.

\section{Current understanding}

At current levels of training, do health care workers understand what 'palliative care' means? Have community workers grasped the concept of palliative care and how do they implement it? In July 2008, an assessment of specific donor-funded programmes across Malawi was carried out to assess current palliative care implementation and future potential. The comments of programme coordinators, health workers and volunteers provided valuable insight into what palliative care currently means to some stakeholders. A wide range of interpretations was evident. In Thyolo; a nurse understood palliative care to be just the same as $\mathrm{CHBC}$, an HIV counsellor coordinator felt that palliative care was broader in scope than $\mathrm{CHBC}$ and a clinical officer understood that palliative care gives patients a positive way of living, and enables them to make informed choices while remaining with their family. Further analysis of responses is provided below.

\section{No idea}

There has been no targeted palliative care sensitisation of programme coordinators and managers who are non-health care workers, despite their key role in service development. This was a significant finding and suggests that health workers may be attempting to provide palliative care services without the support of programme coordinators. Many readily admitted that they have no knowledge of palliative care nor have they heard of morphine to manage severe pain. "T've beard the name, maybe you can assist me", said the Board member of a CHBC programme in Namwera.

The assessment also showed limited understanding of palliative care amongst many $\mathrm{CHBC}$ community volunteers. One volunteer in Karonga offered: "T heard this term during CHBC training. It was mentioned but I have forgotten. I need to revisit my notes". "Our volunteers don't know the term" admitted the technical advisor on a CHBC programme in Chitipa. The assessment was unable to determine the dissemination structure for training in each site, but it suggests that where there are trained health workers', their palliative care knowledge and skills do not filter down to community volunteers.

\section{Care given to people with incurable disease}

Many people interviewed during the assessment understood palliative care to be for people with an incurable disease. A medical assistant in Mponela defined palliative care as "care given to a patient where the illness has no cure". "When one has suffered from a chronic illness and will face the end of life; where there is no hope for recovery, they should die peacefully," said a nurse in Lilongwe.

Although palliative care is indeed beneficial for people at the end of their lives, it is also relevant at the time they are diagnosed with an incurable illness, when they are still healthy. Associating palliative care only with dying and death, rather than with the diagnosis of a life-threatening condition is not accurate, and could result in less or late referrals to much needed services. Palliative care is about life, quality of life, and allowing people to make informed choices about the way they want to live the rest of their life. 


\section{Care given at home or in hospital}

Further confusion arises about where palliative care is offered, and to a large extent it is mistaken as simply a form of community home based care. A nurse interviewed in Ekwendeni described palliative care as "looking after someone who is sick and chronically ill at his/her home and managing problems that may arise". A medical assistant working in Mponela suggested that, "palliative care used to be seen as part of CHBC and now seems to stand alone. We think it is done in health facilities... the organisation is confused'

Palliative care promotes the concept of the patient being encouraged to choose to be where he feels most comfortable. Research indicates that when given a choice and the right support, most patients prefer to be cared for at home. In certain circumstances it is possible for the patient to be fully cared for in his home; where pain relief can be prescribed and administered in the home, and where emotional, practical and spiritual support are skilfully provided by a combination of informal (family and neighbours) and formal carers (health workers and trained volunteers). When this is not possible, referral to the nearest health facility is often the next step. Yet such a referral is only worthwhile when the health facility has the capacity to provide palliative care, referring a patient from the community to a health facility that provides much the same care that is available at home is not the best use of the family's finances and the patients' energy.

\section{Good 'psychosocial' care}

Many people appear to be unclear how the valuable services provided through $\mathrm{CHBC}$ can be enhanced with the addition of palliative care training. Community volunteers and health professionals need to understand the value and limits of their different roles in care provision, so that they can work together to improve quality of life of the patient. In Malawi the culture of $\mathrm{CHBC}$ has become widely recognised and established. $\mathrm{CHBC}$ is typically provided by un-paid women who volunteer to visit and care for the sick in their community. Some aspects of palliative care can be provided by well-trained CHBC volunteers: basic physical care, spiritual and emotional support.

Living while facing death raises real issues which need to be addressed through counselling. This assessment revealed that trained and untrained community volunteers, rarely have counselling skills beyond providing the comfort that friends or neighbours would be able to give. Although many recognised the importance of counselling, all admitted that they seldom approach issues of mortality and loss with their clients and they do not feel they have the counselling skills needed to discuss issues of death and dying. This requires a skilled approach of asking the right questions, learning how to listen and having the confidence and knowledge to work with the raw and extreme emotions that are occasionally encountered. The 'spiritual support' offered by community volunteers often refers to prayers and encouragement for healing and may not encompass asking the patients questions and addressing his personal spiritual concerns.

\section{Emphasis on physical suffering and pain relief}

A nurse in Nkhoma suspected that palliative care is understood by most health care workers as only pain control.
A nurse working in Namwera had completed the introductory training but the only way she could differentiate palliative care from $\mathrm{CHBC}$ was by the relief of pain. A clinical officer in Ekwendeni described palliative care as "clinicians and nurses relieve the pain of somebody chronically ill with cancer or severe pain, bedridden, beyond control by simple care..." This emphasis on pain control leads to a misperception that palliative care is simply CHBC with pain medications added. The holistic and comprehensive nature of palliative care demands that pain assessment and control is one part of the whole package of care.

Having said that, few of the health workers involved in the assessment were familiar with the WHO analgesic ladder as a guide to pain relief. Those who were could not confidently name the drugs at each level. CHBC volunteers do not have the necessary skills to assess pain when a patient is in severe physical pain, and they cannot prescribe the appropriate drugs. There was an unequivocal response in this situation to refer patients to a health worker (at the nearest health facility or beyond) for pain assessment and control. 'Total pain' (an understanding of a holistic approach to pain including physical, social, emotional and spiritual components) was not a concept of which respondents were aware.

\section{A holistic approach}

Most people's understanding of palliative care emphasised either the physical aspect of pain relief, or 'psychosocial' care (varying in depth and quality), but it was reassuring to see that some respondents did have a balanced understanding of the holistic approach that is the heart of palliative care. "When a client receives all necessary support; spiritual, nutritional, medical, material" was the definition given by the coordinator of a programme in Karonga which provides orphan care as well as adult CHBC. A health worker in Chitipa thought that palliative care would involve "giving pain control drugs, providing spiritual counselling and providing counselling and reassurance to both patients and families". It was outside the scope of this assessment to identify whether such understanding should be credited to personal experience, training, or another source.

\section{Giving meaning to words}

Palliative care's focus on quality of life of the patient (rather than finding a cure) is a relatively new and unique approach in Malawi. Palliative care practitioners and promoters believe it is important that patients and their families know what care to ask for and that they are heard and responded to as appropriately as possible. It is important that care providers (family, volunteers and health professionals) understand the scope of what is possible so that they can work together to care for the patient at home or in a health facility. It is therefore extremely important that policy makers and programme coordinators understand the value of this new approach; in order to build skills and source drugs to facilitate the provision of high quality care for those living with incurable illnesses.

The World Health Organisation has defined palliative care as 'an approach that improves the quality of life of patients and their families facing the problems associated with lifethreatening illness, through the prevention and relief of suffering by means of early identification and impeccable 
assessment and treatment of pain and other problems, physical, psychosocial and spiritual.' ${ }^{3}$

Understanding the term 'palliative care' really only matters in as much as it makes a difference to the patients' life. The patient needs to be comfortable, pain-free, emotionally and spiritually supported. The patient needs to be able to ask those around him about his diagnosis and receive accurate information. The patient needs to be able to choose to remain in his home during the course of his illness if he so wishes. As far as possible, the patient and his family need to have their concerns sensitively discussed and competently addressed. This patient-centred, holistic approach must be transferred through effective training methods and styles to care-providers in Malawi.

\section{Reference}

1. Hunt, J. (2008) 'Palliative Care Assessment of Ten Pact Malawi Partner Organisations'. African Palliative Care Association Report for USAID (Unpublished)

2. Ministry of Health (2008) 'Introduction to Palliative Care' Health Care Workers' Training Manual (Unpublished)

3. WHO (2002) Palliative Care [www.who.int/cancer/palliative/definition/ en/]

\section{Palliative Care Association of Malawi: $1^{\text {st }}$ AGM}

\section{Jessica Mackriell}

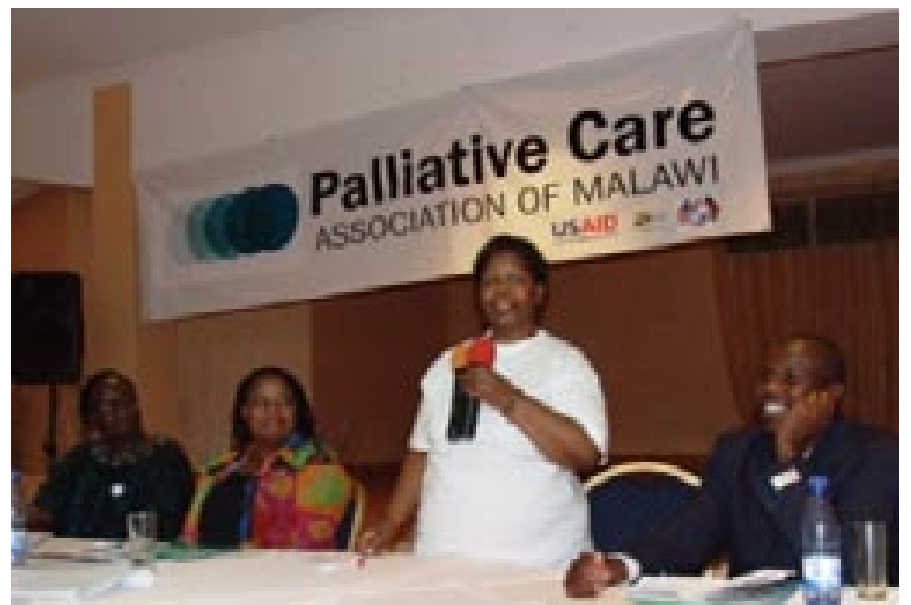

Dr. Mwangi-Powell, Mrs. Musimwa, Matron Kambiya, and Mr. Gombwa

The Palliative Care Association of Malawi (PACAM) is a network of individuals and organisations working together to develop palliative care in Malawi; improving access and building quality. This is achieved strategically through advocacy, training, technical advice and support.

PACAM held its first Annual General Meeting in Lilongwe on 18th October, 2008. With over 200 individual members and 18 member organisations, the Association brings together a significant number of care providers from across the country. The meeting aimed to provide a forum for shared experiences, educational seminars and member feedback.

Sr. Anne Carr, Director of the Interdenominational Pastoral Care Centre and Chair of the PACAM Board of Trustees described how palliative care in Malawi '... addresses the agony and anguish of our people, relatives and above all sons and daughters of God.' Palliative care sites presented their experiences: Tiyanjane Clinic- Blantyre, Ndi Moyo - Salima, and Lighthouse - Lilongwe. A palliative care patient whose pain is being managed with morphine, explained how this has improved his quality of life. Members at the meeting choose between several seminars covering palliative care nursing, community mobilisation, communication, childrens' needs and morphine use.

After lunch the key note speaker - Dr. Faith Mwangi-Powell from the African Palliative Care Association - described the importance of palliative care across Africa and the role that national associations can play. Matron Immaculate Kambiya, the National Palliative Care Coordinator at the Ministry of
Health expressed the commitment of the government to develop palliative care services. Mrs. Kate Musimwa assured PACAM members of continued donor support for palliative care, she represented the US Agency for International Development/Malawi and its cooperating agency Pact Malawi, who kindly funded the meeting.

Directors of PACAM presented the annual report, detailing achievements in the focus areas of drug availability, education, service delivery, networking and organisation development. They explained that they are developing a new strategy which will start in January 2009.

This meeting was an opportunity for care providers to ask questions face-to-face with the Directors of PACAM. Questions explored the association's objectives and how these will provide support to members. People also asked about the possibilities for further training and whether PACAM collaborates with other main stakeholders. They encouraged PACAM to advocate for better palliative care drug availability.

Membership of PACAM is open to anyone with an interest in developing palliative care services in Malawi. There are options for organisational and international membership.

For further information about membership, or for a copy of the annual report please email info.pacam@gmail.com.

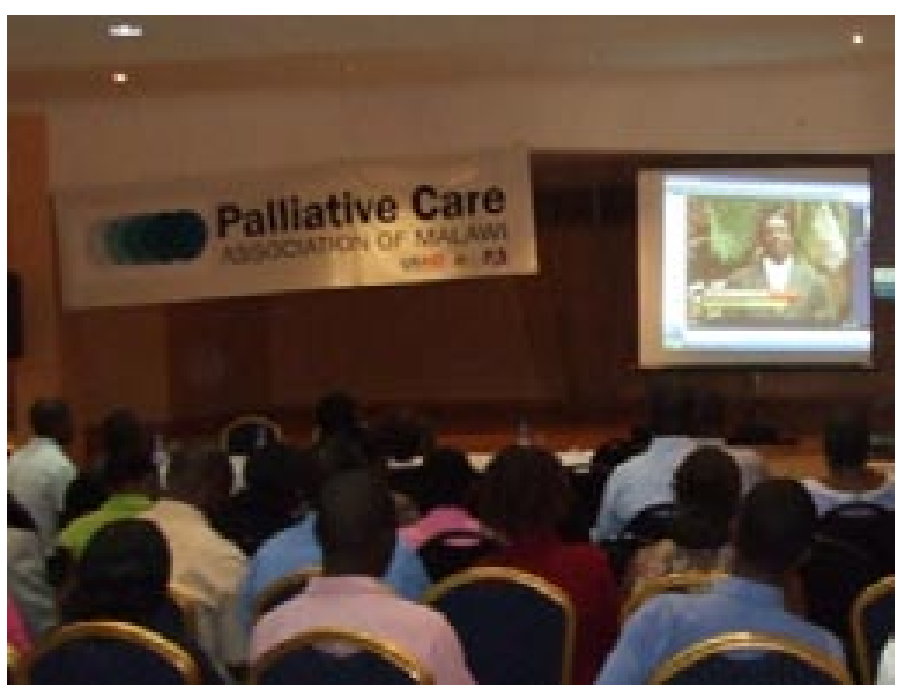

Seminar on morphine use 